\title{
Coagulation and fragmentation dynamics of inertial particles
}

\author{
Jens C. Zahnow, ${ }^{1}$ Rafael D. Vilela,${ }^{2}$ Ulrike Feudel, ${ }^{1}$ and Tamás Tél ${ }^{3}$ \\ ${ }^{1}$ Theoretical Physics/Complex Systems, ICBM, University of Oldenburg, 26129 Oldenburg, Germany \\ ${ }^{2}$ Max Planck Institute for the Physics of Complex Systems, 01187 Dresden, Germany \\ ${ }^{3}$ Institute for Theoretical Physics, Eötvös University, H-1518 Budapest, Hungary
}

\begin{abstract}
Inertial particles advected by many natural and industrial flows undergo coagulation upon collisions and fragmentation if their size becomes too large or if they experience large shear. Here we study this advection-coagulation-fragmentation process in time-periodic incompressible flows. We find that this process approaches an asymptotic, dynamical steady state where the average number of particles of each size class is roughly constant. We compare the steady-state size distributions corresponding to the two fragmentation mechanisms and find that shear-induced fragmentation leads to a distribution which has an invariant functional form, similar to what has been reported to occur in shear-fragmentation experiments in stirring tanks. When only coagulation is taken into account, we observe that a distribution initially peaked on small sizes can eventually develop a second peak at large sizes, comparable with the collisional growth of cloud droplets. Finally, we comment on the robustness of our findings.
\end{abstract}

PACS numbers: 05.45.-a, 47.52.+j, 47.53.+n

\section{INTRODUCTION}

The dynamics of inertial particles in fluid flows is important in several natural and industrial contexts. It has been subject of increasing interest in several disciplines, from dynamical systems [1-8] to atmospheric science [9$11]$ and turbulence $[12,14,15]$. Almost all the works have been devoted to purely advective dynamics of inertial particles. A major reason for this is that the advection dynamics is already very rich, displaying features yet to be understood in their full complexity, as inhomogeneous spatial distributions [16] and multivalued velocity fields $[12,17]$. Interestingly, these very same features yield an increased rate of collisions, the consequences of which are in most cases not explicitly taken into account. Typically one assumes a dilute regime and fully neglects the collisions. In some other works, one keeps track of the collisions numerically without actually addressing the outcome of such events (ghost collisions) $[14,18]$. To our knowledge, only very recent works have addressed effects of collisions on the dynamics of inertial particles $[19,20]$.
In Ref. [19], we have reported our first results on the dynamics of inertial particles coagulating upon collisions and fragmenting under certain conditions. In Ref. [20], the authors considered elastic collisions in a monodisperse system and pointed out the existence of bursts in the spread of the particles out of the attractors of the purely advective dynamics.

In this paper we extend the work of Ref. [19] to different flows and to a broadened parameter set. Our motivation lies primarily on natural phenomena as the collisional growth of cloud droplets [21], sediments in lakes and rivers, and marine snow in the ocean [22]. We consider fragmentation to be of two possible origins. First, particles break up if their size exceeds a certain maximum allowed size. This is motivated by the hydrodynamical instability of large water drops (e.g. cloud drops) settling due to gravity [24]. Second, particles fragment if the shear forces due to the fluid flow are sufficiently large. This mechanism has been reported to be the dominant one in the case of marine snow [31].

At a first glance, one might be tempted to pursue a 
field-theoretical approach, inertial particles, in the framework of which one treats the problem of particle motion as a multiphase flow and then applies the Smoluchowski equation [13] to model coagulation and fragmentation for the particle distribution. The particle velocity may, however, take on several values even at the same location of inertial particles. Due to the presence of such 'caustics' $[12,17,26]$, a field-theoretical approach cannot be well founded. Therefore a study based on an individual tracking of the particles, as is the one presented here, becomes necessary.

Here we consider the fluid flow to be spatially smooth and to have a single macroscopic time scale. We are motivated by flows having coherent (e.g. convective) structures on length scales much larger than the ones at which turbulence plays a major role. The effect of turbulence can then be taken into account as a stochastic perturbation described by an eddy diffusivity [23] at small scales. For simplicity, we neglect this small scale noise in the present work and focus only on the large scale motion of the fluid.

We study the dynamics of the system formed by the advected inertial particles undergoing coagulation and fragmentation in three different fluid flows, as described in Section II. We find that the system tends to approach a steady state where several size classes coexist (Section III). The average number of particles in each size class is roughly constant, with a mild periodic time dependence - with a period identical to the one of the advecting fluid flow. The distribution of particles as well as the mean average size in the steady state depends on the type of fragmentation mechanism taking place. First, when fragmentation occurs solely due to particles exceeding a maximum allowed size, the distribution is in general quite broad. Second, for fragmentation occurring also under sufficiently large shear, the distributions typically decay exponentially fast beyond a certain size class. The distributions depend on the fluid flow for both types of fragmentation. In the case of shear fragmentation, we derive a scaling relation for the average size class in the steady state as a function of the coagulation strength parameter $\gamma$. The size distribution in the steady state has in this case a functional form which does not depend on $\gamma$. In the Discussion we also study the dynamics without fragmentation. In this situation, we observe that a distribution initially peaked on small sizes can eventually develop a second peak at large sizes, a process that has similarities with the collisional growth of cloud droplets [21]. Finally, we show that our results are robust with respect to the number of allowed size classes.

\section{ADVECTION, COAGULATION AND FRAGMENTATION MODEL}

\section{A. Advection}

First, we present the equations of motion for the advection of finite size particles that will be used here. For simplicity we consider heavy spherical aerosols, i.e. particles much denser than the ambient fluid and assume that the difference between their velocity $\dot{\mathbf{x}}$ and the fluid velocity $\mathbf{u}=\mathbf{u}(\mathbf{x}(t), t)$ at the same position is sufficiently small so that the drag force is proportional to this difference (Stokes drag). The dimensionless form of the governing equation for the path $\mathbf{x}(t)=\left(x_{1}(t), x_{2}(t)\right)$ of the center of mass for such heavy aerosols subjected to drag and gravity reads in this case as [32-34]:

$$
\ddot{\mathbf{x}}=\frac{1}{\tau}(\mathbf{u}(\mathbf{x}(t), t)-\dot{\mathbf{x}}-W \mathbf{n}),
$$

where $\mathbf{n}$ is a unit vector pointing upwards in the vertical direction. Throughout this paper we consider the vertical direction along the axis $x_{2}$. Under the assumption that the density ratio $\rho_{f} / \rho_{p} \ll 1$, the particle response time $\tau$ can be written in terms of the density $\rho_{p}$ of the particle, the radius $a$ of the aerosols, the fluid's dynamic viscosity $\eta$, and the characteristic length $L$ and velocity $U$ of the flow as $\tau=\left(2 a^{2} \rho_{p} U\right) /(9 \eta L)$. We note that the response time $\tau$ is nothing but the Stokes number 
which can be written in our case as $\tau=\tau_{p} / T$ where $\tau_{p}$ is the particle's dimensional Stokesian relaxation time and $T$ is the characteristic time of the flow. The dimensionless settling velocity in a medium at rest is given by $W=\left(2 a^{2} \rho_{p} g\right) /(9 \eta U)$.

Every particle produces perturbations in the flow that decay at least inversely proportional to the distance from the particle [37, 38]. Here we assume a dilute regime, where the local concentration of particles is low enough, so that particle-particle interaction can be neglected [43] unless particles come into direct contact.

The assumption that the particle radii $a$ are small also means that the feedback from the particle motion on the flow will be small as well [34] and is therefore neglected in the following.

\section{B. Coagulation}

Second, we present a model for the coagulation of finite size particles.

The smallest particles considered will in the following be called primary particles. These primary particles can combine to form larger particles, called coagulates. Coagulation takes place upon collision. All particles are assumed to consist of an integer number of these primary particles, i.e. the primary particles can never be broken up. The number $\alpha$ of primary particles in a coagulate is called the size class index. We consider $n$ different size classes, i.e. coagulates can consist of a maximum of $n$ primary particles. A coagulate of size class $\alpha$ has a radius $a_{\alpha}=\alpha^{1 / 3} a_{1}$, where $a_{1}$ is the radius of the primary particles. The response time is $\tau_{\alpha}=\left(a_{\alpha} / a_{1}\right)^{2} \tau_{1}=\alpha^{2 / 3} \tau_{1}$ and the settling velocity in still fluid is $W_{\alpha}=\alpha^{2 / 3} W_{1}$. Here $\tau_{1}$ and $W_{1}$ are the response time and the settling velocity for the primary particles, respectively. The largest coagulates therefore have a radius $a_{n}=n^{1 / 3} a_{1}$. We note that particles of different sizes have different parameters $\tau_{\alpha}$ and $W_{\alpha}$ and therefore follow the flow with different parameters in the equation of motion (1).
We define a collision of two particles if the centers of the particles, say of radius $a_{i}$ and $a_{j}$, come closer than a distance $d=a_{i}+a_{j}$. In that case the particles coagulate and form a larger particle. Mass conservation requires the radius of the new particle to be $a_{\text {new }}^{3}=a_{i}^{3}+a_{j}^{3}$. For the size class index this implies a linear rule, $\alpha_{\text {new }}=\alpha_{i}+\alpha_{j}$, which determines the new response time and settling velocity via $\tau_{\alpha_{n e w}}=\alpha_{n e w}^{2 / 3} \tau_{1}$ and $W_{\alpha_{\text {new }}}=\alpha_{\text {new }}^{2 / 3} W_{1}$, respectively.

The velocity of the new particle follows from momentum conservation. The position of the new coagulate is the center of mass of the two old particles.

\section{Fragmentation}

Third, we present a model for the fragmentation of particles. Primary particles cannot be broken up. In the following, we will compare two different fragmentation rules.

(i) Size-limiting fragmentation: If a particle becomes larger than the maximum radius $a_{n}$, it is broken up into two smaller fragments (binary fragmentation) whose radii are chosen randomly, from a uniform distribution between $a_{1}$ and half the original radius. If any fragment is larger than $a_{n}$ this process is repeated, until no fragment exceeds $a_{n}$.

(ii) Shear fragmentation takes place when the hydrodynamical force $F_{h y d}$ acting on the particle exceeds the forces $F_{\text {coag }}$ holding the coagulate together by a certain factor. The criterion for breakup can therefore be expressed as [36]

$$
F_{h y d} / F_{\text {coag }}>\tilde{\gamma}
$$

where $\tilde{\gamma}$ is a constant.

The hydrodynamical force in this case is proportional to the local velocity gradients in the flow. For solid spherical particles the hydrodynamical forces are usually far too weak to break up these particles. However, for liquid particles, e.g. water drops moving in air, shear forces are 
generally able to break up the particles. For liquid spherical particles (drops) in the size range where viscous forces dominate, Taylor [39] and later Delichatsios [40] derived an expression for the critical velocity difference $\Delta u$ across the drop required for breakup. They treated a drop as a vibrating spring-mass system and calculated the breakup velocity gradient under the condition that the characteristic time of drop deformations is small compared to the time where this velocity gradient occurs. Their result can be written in our notation as

$$
\frac{\Delta u}{a_{\alpha}} \propto \frac{\sigma}{\mu_{p} a_{\alpha}},
$$

where $\sigma$ and $\mu_{p}$ is the surface tension and the viscosity of the drop, respectively.

For our purposes here this condition can be written with a single parameter as

$$
\frac{\Delta u}{a_{\alpha}}=\gamma\left(\frac{a_{1}}{a_{\alpha}}\right)=\gamma \alpha^{-1 / 3}
$$

where $\gamma$ is a constant, the coagulate strength parameter (the same quantity is called stickiness in [19]). The radius has been normalized with the radius of a primary particle. If the maximum velocity difference across the radius of the drop exceeds the threshold value given by Eq. (4), the particle is broken up into two smaller fragments (binary fragmentation) in the same way as for size-limiting fragmentation.

At the instant of both coagulation and fragmentation there is a sudden change in the dynamics: the number of particles changes in 2 or 3 among the $n$ available dynamical systems defined by the size classes.

\section{Fluid flows}

For convenience, we treat the case where the fluid flow is two-dimensional, therefore the phase space of the advection dynamics is 4-dimensional. We choose three simple paradigmatic flow situations with different characteristics to indicate the generality of our results.

All flow domains are spatially periodic, with a characteristic length $L$. More specifically, the flows are (a) a convection cell flow with moving vortex centers (in the following referred to as the moving convection flow), (b) a convection cell flow with fixed vortices (referred to as the fixed convection flow), (c) a sinusoidal shear flow.

The two convection cell flows (a) and (b) consist of a regular pattern of vortices, or roll cells. Flow (b) was first introduced by Chandrasekhar [46] as a solution to the Rayleigh-Bénard problem and since then it has been used in the context of different theoretical studies $[1,29,41,49]$. The moving convection flow (a) is a slightly modified version, with moving vortex centers, to yield a more realistic chaotic regime for the particle motion. Convection flows are chosen because they contain vortices (convection cells) and uprising/sinking regions, which are characteristic features of realistic flows often found in nature. The flows are defined by the velocity field

(a) moving convection flow

$\mathbf{u}\left(x_{1}, x_{2}, t\right)=\left[1+k_{1} \sin \left(\omega_{1} t\right)\right]\left(\begin{array}{c}\sin \left(2 \pi \hat{x_{1}}\right) \cos \left(2 \pi \hat{x_{2}}\right) \\ -\cos \left(2 \pi \hat{x_{1}}\right) \sin \left(2 \pi \hat{x_{2}}\right)\end{array}\right)$

where $\hat{x_{1}}=x_{1}+k_{2} \sin \left(\omega_{2} t\right)$ and $\hat{x_{2}}=x_{2}+k_{2} \cos \left(\omega_{2} t\right)$. The parameters $k_{1}=2.72$ and $\omega_{1}=\pi$ are the amplitude and the frequency of the periodic forcing of the flow, respectively. $\quad k_{2}=1 /(2 \pi)$ and $\omega_{2}=\pi / 4$ determine the amplitude and the frequency of the periodic motion of the centers of the vortices in the flow. The period of the flow is $T=2$ and the characteristic length and velocity are $L=1$ and $U=1$.

(b) fixed convection flow with the same equation for the flow as in (a), but with the $k_{2}=0$.

The sinusoidal shear flow (c) consists of alternating horizontal and vertical velocity components, where each velocity component consists of two plateaus in time. It was introduced in Refs. [47, 48] and has been used many times in chaotic advection studies. Here we consider a time-continuous version (see [28]) defined by: 
(c) sinusoidal shear flow

$\mathbf{u}\left(x_{1}, x_{2}, t\right)=0.5\left(\begin{array}{c}(1+\tanh (\beta \sin (2 \pi t))) \sin \left(2 \pi x_{2}\right) \\ (1-\tanh (\beta \sin (2 \pi t))) \sin \left(2 \pi x_{1}\right)\end{array}\right)$,

where the parameter $\beta$ describes how rapidly the transition between two values, a zero and a nonzero velocity, takes place for each velocity component. The typically used value $\beta=20 / \pi$ corresponds to a very rapid transition.

The period of the flow is $T=1$ and the characteristic length and velocity are $L=1$ and $U=1$.

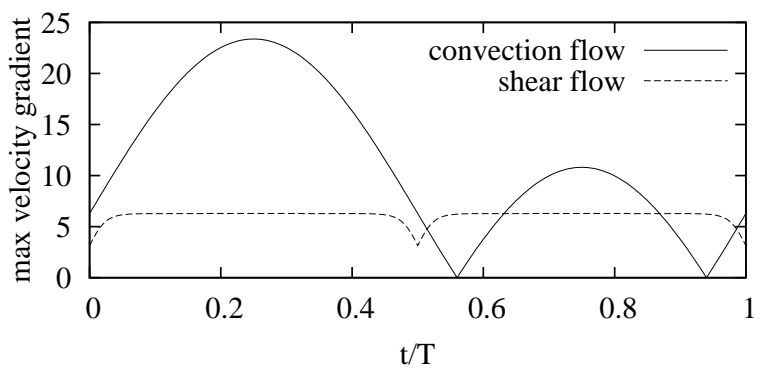

FIG. 1: Maximum of the velocity gradient $\operatorname{grad} \mathbf{u}\left(x_{1}, x_{2}, t\right)$ vs. time for the two different flows described by Eqs. (5) and (6).

The fluid flows are laminar and time-periodic, but the dynamics of the inertial particles moving in these flows can be chaotic.

To emphasize the difference between the flows, Fig. 1 shows the maximum of the velocity gradient vs. time for the convection flows (there is no difference between the moving and the fixed convection flow) and the sinusoidal shear flow. The difference in magnitude and also in the temporal evolution between these two flows is clearly visible, indicating a possibly very different behavior with respect to shear fragmentation.

\section{E. Numerical implementation}

After presenting the model, we describe some details about the implementation. In the bulk of the paper we consider $n=30$ size classes. The primary particles considered here have dimensionless radius $a_{1}=5 / 30^{1 / 3} \times$ $10^{-5}$, mass $m_{1}=\rho_{p} \frac{4}{3} \pi a_{1}^{3}$, response time $\tau=1 / 55$ and settling velocity $W_{1}=3.2 \tau$.

The number of coagulates $N(t)$ changes over time due to coagulation and fragmentation leading to a distribution of coagulates of different radii in the flow, but the total mass $M=\sum_{i=1}^{30} \alpha_{i}(t) m_{1}$ remains constant during any simulation.

As initial condition we take $10^{5}$ primary particles and no larger particles. Furthermore, particles are uniformly distributed over the $1 \times 1$ unit cell of the configuration space. This choice fixes the total mass of the system to be $M=10^{5} m_{1}$. The initial particle velocity matches that of the fluid at their position in all cases. All three aspects, advection, coagulation, and fragmentation that define the whole dynamics can be modelled separately.

The simulation is based on the following ingredients:

1. All particles move in the flow over some time step $d t$ according to Eq. (1). This integration time step $d t$ needs to be chosen small enough to allow for the detection of every collision. We emphasize again at this point that each coagulate size is characterized by different values of $\tau_{\alpha}$ and $W_{\alpha}$, so that the motion of particles of different size is governed by the same equations but with different parameters.

After each time step $d t$ there is an interaction between particles in the form of coagulation if they are too close to each other. As a result, an unrealistic clustering of particles (an infinitely large particle density) cannot be formed in contrast to cases where the center of mass motion of the particles is considered only (see e.g. [26]). Our experience shows that a choice $d t=T / 20$ is sufficiently small for the conditions considered here.

Because of the spatial periodicity of the flow, the particle dynamics is folded back onto the $1 \times 1$ unit cell, using periodic boundary conditions (see e.g. 
[29, 49]). Without folding back, particles heavier than the fluid do not stay in a single unit cell of the flow. In particular, they are not suspended [16]. Instead, particles generally fall downwards into a vertically adjacent cell. This means that folding the dynamics of the particles back onto the unit cell is only a convenient way to visualize an infinitely extended system. If particles are initially distributed homogeneously over the whole cell, the total particle mass $M$ remains the same over time and therefore even if coagulation and fragmentation are included, it is sufficient to restrict our studies to one unit cell with periodic boundaries.

2. Particles coagulate if their distance is smaller than the sum of their radii. Computationally, the coagulation proccess is the most costly component of the simulation. In particular, the naive approach to calculate which particles are colliding at a given time step involves looping over all pairs of particles and therefore scales as $O\left(N^{2}\right)$ for a single time step, where $N$ is the number of particles. Therefore, here a link-cell algorithm [27] is used to compute the distance between particles. The configuration space is divided into grid cells of size $\epsilon$, where each grid cell stores information on which particles it contains. After each time step this information is updated, to reflect changes in the particle positions. The looping over particle pairs to calculate their distance is then done only over particles in a given grid cell and the neighboring cells. If the grid cell size $\epsilon$ is small enough (but sufficiently larger than the largest possible particle size, $a_{30}$ ) the link-cell algorithm scales as $O(N)$ and is thus much faster than the naive approach.

3. Coagulates can fragment either due to size-limiting fragmentation or due to shear fragmentation.

(a) Size-limiting fragmentation: If the coagulate size $\alpha$ exceeds the predefined maximum size, which is in the following fixed at $n=30$ unless mentioned otherwise, the coagulate is broken up.

(b) Shear fragmentation: If the shear at the position of the coagulate exceeds a critical value, determined by Eq. (4) the coagulate breaks up. Normally, the velocity differences in all directions across a coagulate would have to be calculated and their maximum would have to be found to determine the maximum shear acting on the coagulate. Due to the symmetry of the flows chosen here, the maximum velocity difference is always either between $\left(x_{1}-a_{\alpha}, x_{2}\right)$ and $\left(x_{1}+a_{\alpha}, x_{2}\right)$ or between $\left(x_{1}, x_{2}-a_{\alpha}\right)$ and $\left(x_{1}, x_{2}+a_{\alpha}\right)$, i.e. in the direction of one of the coordinate axes, therefore only these values have to be calculated. Shear fragmentation is always applied together with size-limiting fragmentation to keep the maximum number of occurring size classes fixed at $n$.

Whatever rule is applied, the result is the reversed process of coagulation: two new particles are formed from an old one with the size class indices: $\alpha_{i, n e w}+\alpha_{j, n e w}=\alpha_{o l d}$. As indicated earlier, $\alpha_{i, n e w}$ can take on any value between $\alpha_{1}$ and $\alpha_{n / 2}$ with equal probability. The centers of the new particles are placed along a line segment in a random direction so that the distance $d$ between the particle centers equals the sum of their radii, i.e. $d=a_{i}+a_{j}$, and the center of mass remains unchanged. Momentum is conserved. For simplicity we assume that the new particles have the same velocity as the old one. 


\section{SIMULATION RESULTS}

In this section we show simulation results using the model described above and compare the influence of the different flows, and the effect of size-limiting fragmentation and shear fragmentation.

Before presenting any results for the complete model, it is worth showing the attractors for the non-interacting problem in the different flows. Figs. 2, 3 and 4 show the stroboscopic section (taken with the period $T$ of the flow) of the attractors for the flows (a)-(c) projected onto the plane of the coordinates for four different size classes. (a)

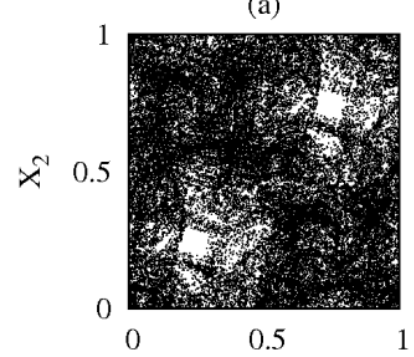

(c)

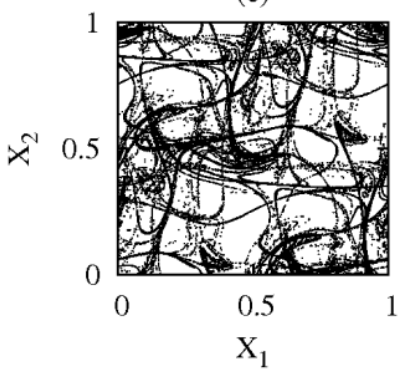

(b)

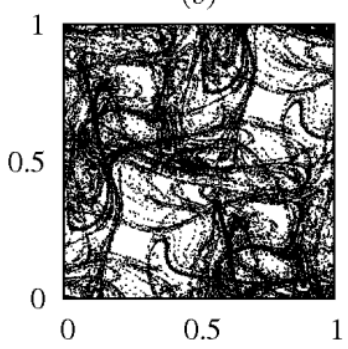

(d)

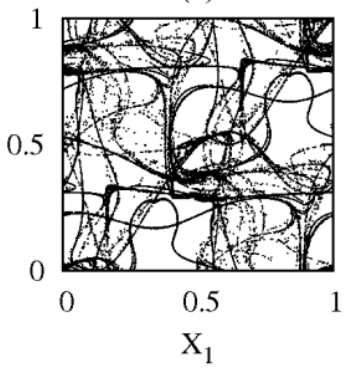

FIG. 2: Stroboscopic sections of the attractors of Eq. for the moving convection cell flow (Eq. (5)) projected onto the configuration space for particle size classes (a) $\alpha=1$, (b) $\alpha=5$, (c) $\alpha=15$ and (d) $\alpha=30$. All attractors are chaotic.

For the moving convection flow and the sinusoidal shear flow the degree of clustering of the particles in the attractors, quantified by their fractal dimension, decreases monotonically with the size class. The parameter region is chosen in such a way that the attractors are either area filling or fractal with dimension smaller than 2, which we consider to be closer to a realistic situation than for example fixed point attractors. (a)

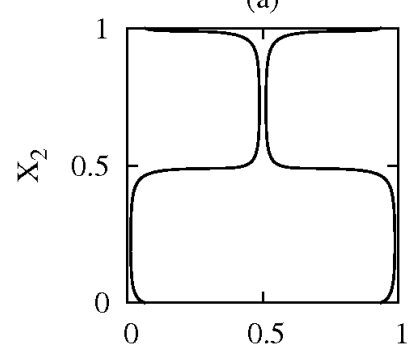

(c)

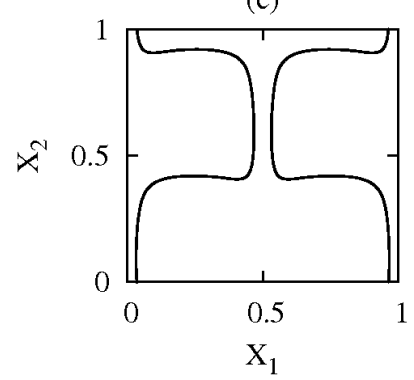

(b)

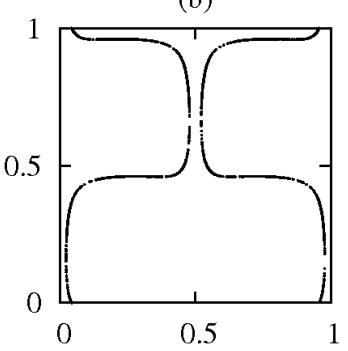

(d)

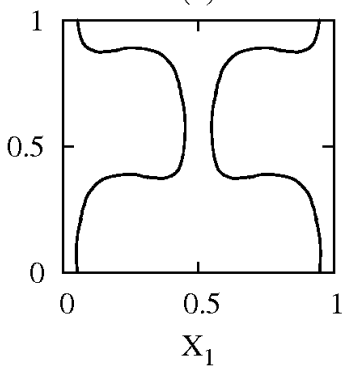

FIG. 3: Stroboscopic sections of the attractors of Eq. (1) for the fixed convection flow $\left((5)\right.$ with $\left.k_{2}=0\right)$ projected onto the configuration space for particle size classes (a) $\alpha=1$, (b) $\alpha=5$, (c) $\alpha=15$ and (d) $\alpha=30$. All attractors are quasiperiodic.

It is worth noting that the finding of [26], according to which the attractors densely fill in the space of the flow for sufficiently large response times, is not valid here, which we attribute to the presence of gravity and the time periodicity of our flows. To provide a contrast, the fixed convection flow has only quasiperiodic attractors in the chosen parameter region, i.e. smooth curves in the stroboscopic section.

Next, we look at the full dynamics, with advection, coagulation and fragmentation as described in the previous section. A useful measure to follow the time evolution of the particle size distribution is the average size class index $\langle\alpha(t)\rangle=\sum_{i=1}^{30} \alpha_{i} N_{\alpha_{i}}(t) / N(t)$, where $N_{\alpha_{i}}$ denotes the number of particles in size class $\alpha_{i}$.

Fig. 5 shows this index for the different flows and the different fragmentation rules. In all flows we find convergence to an asymptotic steady state. Initially, coagulation leads to a fast increase in the average particle size class, independent of the fragmentation rules. Then frag- 
(a)

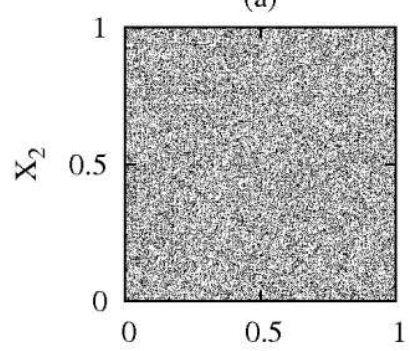

(c)

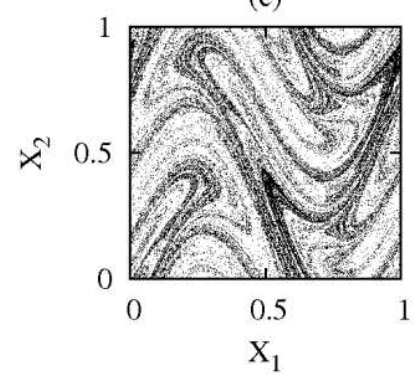

(b)

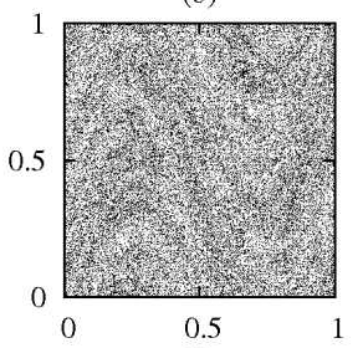

(d)

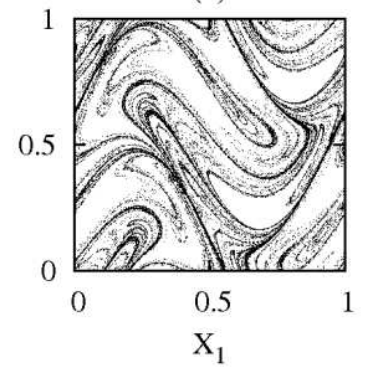

FIG. 4: Stroboscopic sections of the attractors of Eq. for the sinusoidal shear flow (Eq. (6)) projected onto the configuration space for particle size classes (a) $\alpha=1$, (b) $\alpha=5$, (c) $\alpha=15$ and (d) $\alpha=30$. All attractors are chaotic and (a) and (b) are area filling.

mentation sets in and a balance between coagulation and fragmentation is reached, with an asymptotic average coagulate size $\alpha_{\infty}=\lim _{t \rightarrow \infty}\langle\alpha(t)\rangle$ that depends on the fragmentation rule and the different flows. The difference in the time it takes to reach the steady state comes mainly from the different coagulation probabilities in the different flows. The fixed convection flow takes the shortest time to reach the steady state, because the attractors are covering much less area than in the other cases, therefore leading to higher local concentrations of particles.

We note that the shear forces in the convection flows and the sinusoidal shear flow have a different magnitude, as seen in Fig. 1. Therefore three different values of the coagulate strength parameter $\gamma$ need to be chosen to yield a size distribution whithin the range of the 30 allowed size classes. For the convection flows the coagulate strength $\gamma$ needs to be approximately a factor 3 larger than for the sinusoidal shear flow.

In a real system similar particles would of course have a (a) - moving convection flow

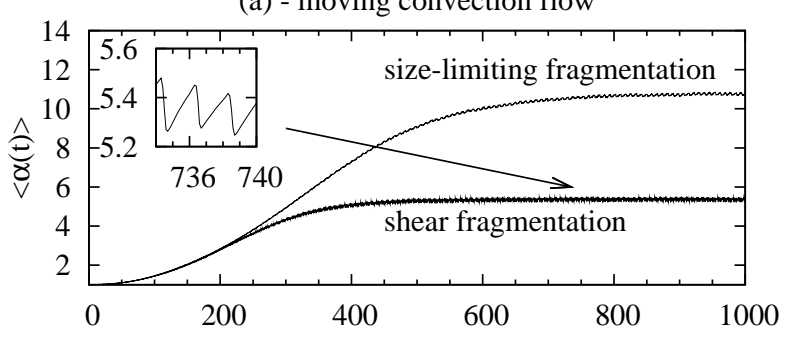

(b) - fixed convection flow

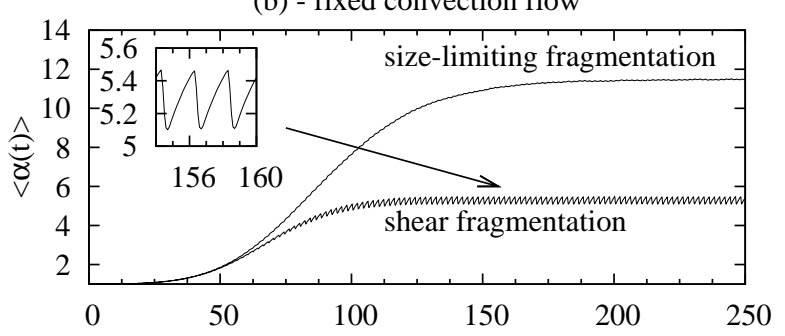

(c) - sinusoidal shear flow

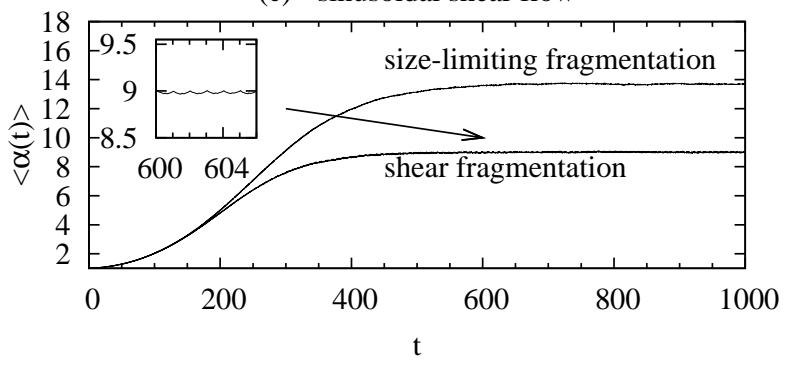

FIG. 5: Average size class vs. time for size-limiting fragmentation (upper curve) and shear fragmentation (lower curve) for (a) moving convection cell flow, (with $\gamma=50$ for shear fragmentation), (b) fixed convection cell flow, (with $\gamma=45$ for shear fragmentation), (c) sinusoidal shear flow (with $\gamma=17$ for shear fragmentation). The insets show the periodic change in the average size class that result from the period of the shear for the moving convection cell flow and the fixed convection cell flow.

similar coagulate strength, no matter what flow they are in. Because in a real system there is no limitation to the number of available particle size classes, the particle size distribution would then be simply shifted to a different position depending on the magnitude of the shear forces in the flow.

More important is the fact that for the convection flow the shear force oscillates smoothly with time, while for the sinusoidal shear flow the shear remains almost constant over time, except for a small 'dip' when the direc- 
tion of the velocity field changes (cf. Fig. 1). In the case of the two convection flows with shear fragmentation, the asymptotic average size class $\alpha_{\infty}$ therefore still oscillates with the same period as the shear forces in the flow. This is clearly visible in the insets in Figs. 5(a) and (b).

For the sinusoidal shear flow with shear fragmentation the amplitude is much smaller because the shear stays almost constant over time. For all flows with size-limiting fragmentation the value of the asymptotic coagulate size class $\alpha_{\infty}$ is almost constant over time (see inset in Fig. $5(\mathrm{a}))$ due to the fact that the maximum size class is the same for all times.

The value of $\alpha_{\infty}$ of size-limiting fragmentations is different in the flows, due to the different coagulation and fragmentation probabilities. However, the qualitative behavior remains the same.

When investigating cases with different total mass $M$, we find that for size-limiting fragmentation $\alpha_{\infty}$ is, in a broad range, independent of $M$. For shear fragmentation with $M<3 \times 10^{6} m_{1}, \alpha_{\infty}(M)$ increases approximately linearly with $M$, while for higher values a saturation of $\alpha_{\infty}(M)$ sets in, which is due to the fact that size-limiting fragmentation is also present in this scenario.

By considering other initial particle size distributions than mentioned above, for example any single size class with $\alpha>1$ or a uniform distribution of sizes, and keeping the total mass $M$ fixed, the asymptotic state is found for each flow and for both fragmentation rules (where it applies) to be independent of the chosen initial distribution. Nonetheless, for shear fragmentation the asymptotic state does depend on the value of the coagulate strength $\gamma$.

To illustrate this dependence of the steady state on the coagulate strength $\gamma$, Fig. 6 shows how $\alpha_{\infty}$ changes, at a fixed $M$ for the different flows.

A drastic increase of $\alpha_{\infty}(\gamma)$ can be observed in the interval $40<\gamma<75$ for the moving convection flow, $40<\gamma<60$ for the fixed convection flow and $13<\gamma<$ 20 for the sinusoidal shear flow. These are the intervals (a) - moving convection flow

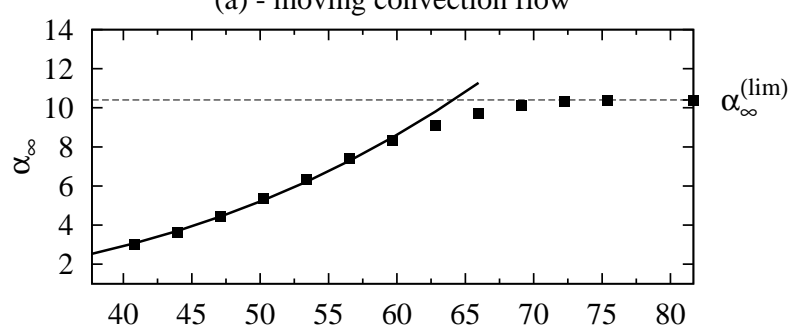

(b) - fixed convection flow

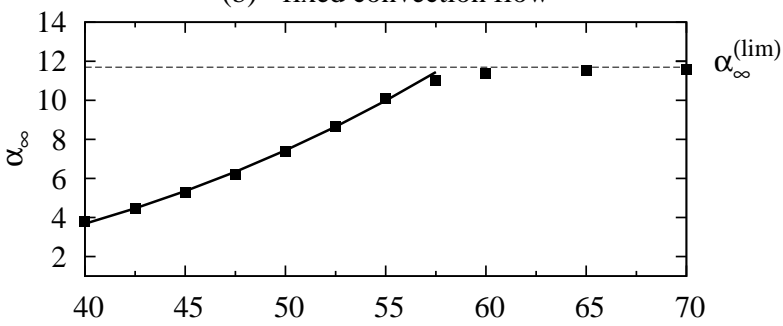

(c) - sinusoidal shear flow

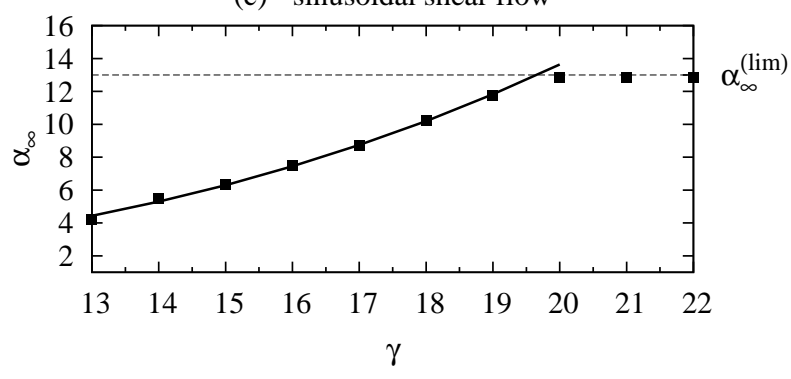

FIG. 6: Asymptotic average size class index $\alpha_{\infty}$ as a function of the coagulate strength parameter $\gamma$. Squares: numerical results, solid line: fit by $\alpha_{\infty}=c_{1}+c_{2} \gamma^{3}$ (see Eq. (8)). (a) moving convection cell flow $\left(c_{1}=0.521, c_{2}=3.7 \times 10^{-5}\right)(\mathrm{b})$ fixed convection cell flow $\left(c_{1}=-0.256, c_{2}=6.2 \times 10^{-5}\right)$ and (c) sinusoidal shear flow $\left(c_{1}=0.945, c_{2}=1.6 \times 10^{-3}\right)$.

for each flow where, at the chosen value of $\alpha_{\max }$, shear fragmentation dominates and size-limiting fragmentation only plays a minor role.

It is clear that $\alpha_{\infty}$ increases with $\gamma$, because particles become more resistant to shear. The exact functional relationship is however not so obvious. A first qualitative estimate of the shape of this $\alpha_{\infty}(\gamma)$ curve can be derived by assuming that over one period of the flow the particles experience an 'average shear'

$$
\bar{G}=\frac{1}{T} \int_{0}^{T} d t \int_{D} d \mathbf{x} p(\mathbf{x}, t) G(\mathbf{x}, t),
$$

where $G(\mathbf{x}, t)$ is the modulus of the local velocity gradi- 
ent, $p(\mathbf{x}, t)$ is the distribution of particles and $D$ is the unit square domain. From Eq. (4) we then get for the average critical size at this velocity gradient

$$
\bar{\alpha}_{c r i t}=\bar{G}^{-3} \gamma^{3}
$$

Particles that exceed this size will therefore typically break up during one period of the flow. Since particles break into two parts due to shear, the average size would then be $\alpha_{\infty} \geq \bar{\alpha}_{\text {crit }} / 2$. The average shear $\bar{G}$ is, however, somewhat complicated to estimate. It would have to be calculated as a mean over the positions of all particles in the flow at a given time. Additionally, how larger than the critical size particles get before they break up depends on the coagulation probabilities, and therefore also on the local concentrations of particles. The exact dependency of $\alpha_{\infty}(\gamma)$ is therefore not easily calculated. What can be seen from Eq. (8) is however that the average size is expected to scale with $\gamma$ as

$$
\alpha_{\infty} \propto \gamma^{3}
$$

even if the proportionality factor varies greatly for different flows and particle distributions. This dependence is expected to hold for all values of $\gamma$ and $\alpha_{\infty}$, where shear fragmentation dominates. A fit with Eq. (9) for the different flows is shown in Fig. 6 and for lower values of $\gamma$ the fits agree very well with the simulation results. It can be seen that for higher values of $\gamma$, when size-limiting fragmentation becomes important, the $\alpha_{\infty}(\gamma)$ curves deviate from this estimate and converge towards the limiting value $\alpha_{\infty}^{(\mathrm{lim})}$ (see Fig. 6).

After studying average quantities we investigate the size distributions, i.e. how the number of particles of each size class looks like for the different fragmentation rules, and address the question of what is the role of the flows.

Figure 7 shows histograms of the particle size distribution for shear fragmentation and size-limiting fragmentation for the different flows. It can be seen that all size distributions have their maxima at the smallest size class
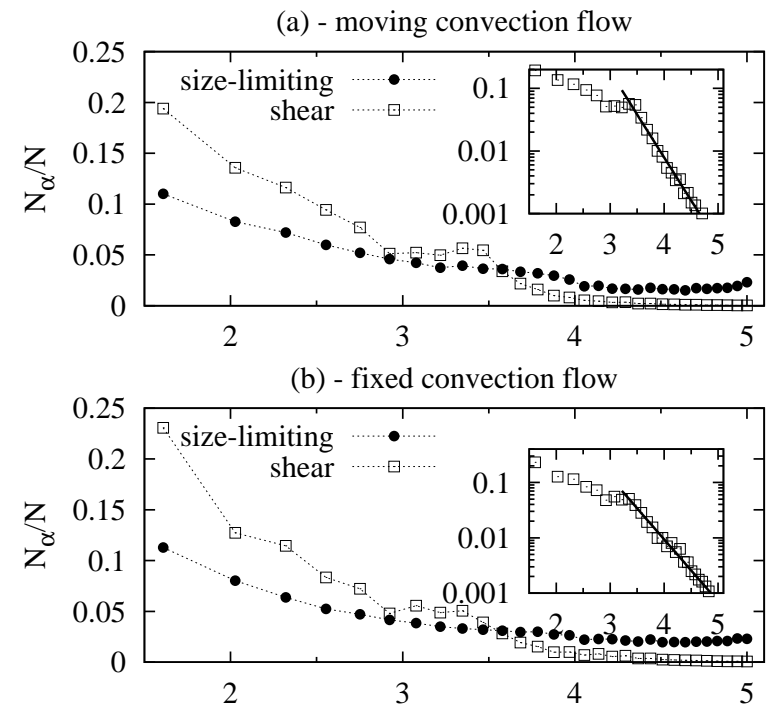

(c) - sinusoidal shear flow

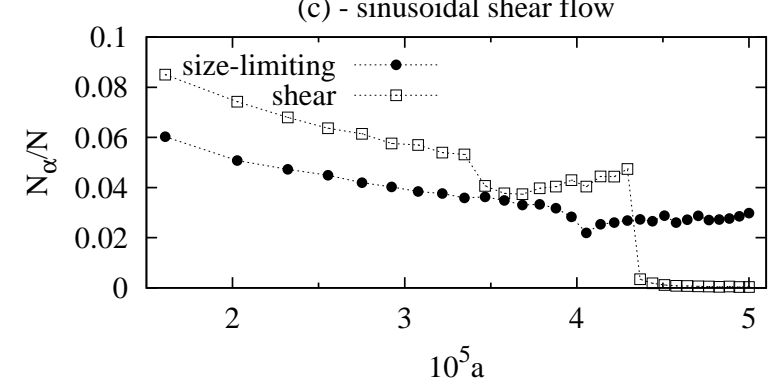

FIG. 7: Histogram of the particle size distribution in steady state for size-limiting fragmentation and shear fragmentation with (a) moving convection cell flow, (with $\gamma=50$ for shear fragmentation), (b) fixed convection cell flow, (with $\gamma=45$ for shear fragmentation), (c) sinusoidal shear flow (with $\gamma=17$ for shear fragmentation). The insets show the exponential tail of the size distributions for shear fragmentation in the case of the moving convection cell flow and the fixed convection cell flow.

and decay for larger size classes. For size-limiting fragmentation the distribution never drops to zero for any flow.

For shear fragmentation the particle size distribution tends to zero beyond a certain size class. In the case of the convection flows the size distribution has a long tail towards larger size classes that decays exponentially. By contrast, for the sinusoidal shear flow the size distribution has a second, smaller peak (in addition to the maximum at the smallest size class) and then drops off 
sharply towards zero beyond that point. Here this sharp drop off occurs due to the almost constant level of shear in the flow, which leads to a clearly defined maximum size for the particles.

(a) - shear fragmentation
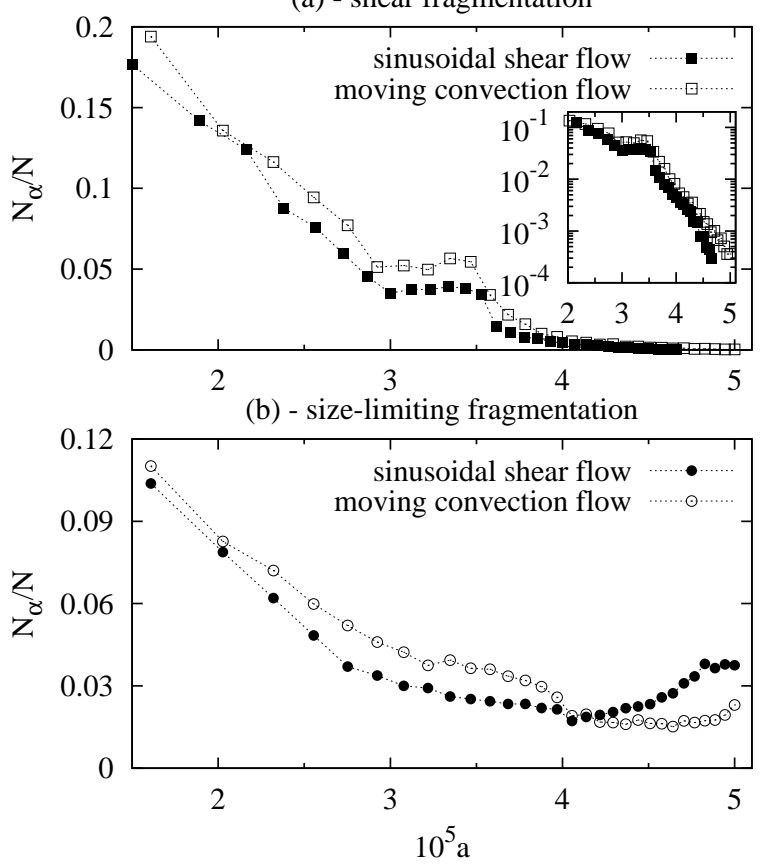

FIG. 8: Histogram of the particle size distribution in steady state for the moving convection cell flow $(\gamma=50)$ and the smoothed out sinusoidal shear flow $(\gamma=8.5, \beta=\pi / 20)$ for (a) size-limiting fragmentation and (b) shear fragmentation.

That this difference is indeed mainly due to the different time evolution of the shear forces in the flow, and does not depend e.g. on the detailed characteristics of the particle motion, can be seen by adjusting the flow parameters. Decreasing the parameter $\beta$ for the sinusoidal shear flow to a much smaller one, e.g. $\beta=\pi / 20$ results in a much smoother transition between the two plateaus for the velocity components. As a consequence we obtain two sinusoidal peaks per period for the shear forces, similar to what happens in the convection flow (cf. Fig. 1), except that for the sinusoidal shear flow both peaks have the same height. For an appropriate choice of $\gamma$, so that the average size classes match, it can be seen that the shape of the particle size distributions for both flows has become almost identical (Fig. 8(a)). Even though the actual motion of the particles is still very different in the two flows, once the shear forces exhibit a similar temporal behavior (in this case all that is required is that the shear in both flows changes comparably smoothly over time) the size distributions are almost identical. Once again we note that the coagulate strength $\gamma$ needs to be different for both flows when trying to get overlapping particle size distributions because the amplitudes of the shear forces are still different. Additionally, this tuning of the parameters also affects the result for size-limiting fragmentation, as can be seen in Fig. 8(b). The size distribution in the case of the sinusoidal shear flow has transformed from an almost homogeneous distribution to one much closer resembling the result for the moving convection flow, even though there is still some difference in the tail of the distribution.

For each flow there is a certain range of the coagulate strength parameters $\gamma$ where the size distribution for shear fragmentation is "fully developed". By this we mean that $\gamma$ is large enough so that a sufficiently large fraction of particles has left the smallest size class, but $\gamma$ is small enough so that break-up due to sizelimiting fragmentation does not play a significant role. For the moving convection flow this range is approximately $40<\gamma<60$, for the fixed convection flow this is approximately $40<\gamma<50$ and for the sinusoidal shear flow the range is approximately $13<\gamma<19$ (cf. Fig. $6)$. In this intermediate $\gamma$ range, where the particle size distribution is fully developed, a scaling form

$$
\frac{N_{\alpha}}{\max \left(N_{\alpha}\right)}=f\left(\frac{a}{\langle a\rangle}\right)
$$

is found to hold, where $\langle a\rangle$ represents the average radius. Note that the form of the size distribution is independent of $\gamma$. All distributions in this parameter range collapse then onto a single master curve.

While this scaling form is independent of the parameters of the coagulation and fragmentation process, it can 
(a) - moving convection flow

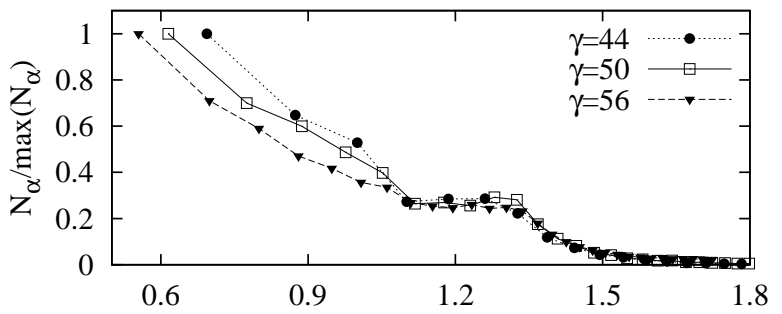

(b) - fixed convection flow

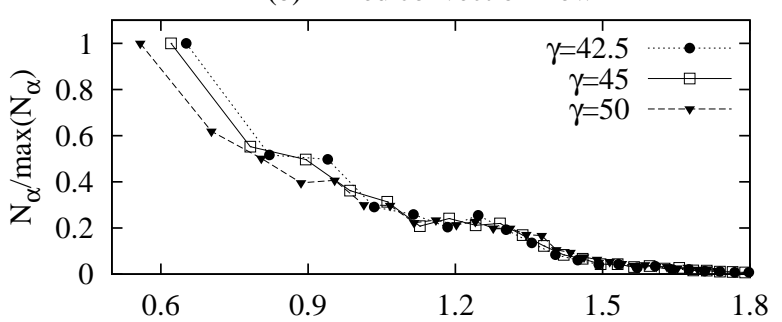

(c) - sinusoidal shear flow

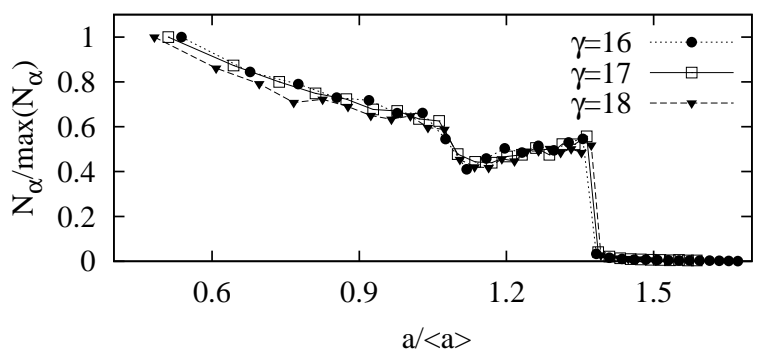

FIG. 9: Normalized number of particles versus relative radius in steady state for different values of the coagulate strength parameter $\gamma$ for (a) moving convection cell flow, (b) fixed convection cell flow and (c) sinusoidal shear flow $(\beta=20 / \pi)$.

be different for different flows. More specifically, the scaling form $f$ changes when the shear force distribution of the flows varies very differently over time.

\section{DISCUSSION}

We discussed the formation of a steady state size distribution in a coagulation fragmentation process of particles advected by different flows. We note that our findings are robust against several changes of the model. The effect of the number of new particles formed by fragmentation has been considered. For instance, the distributions of particles for ternary fragmentation are similar to the ones for binary splitting and only show a slight shift towards smaller size classes [51].

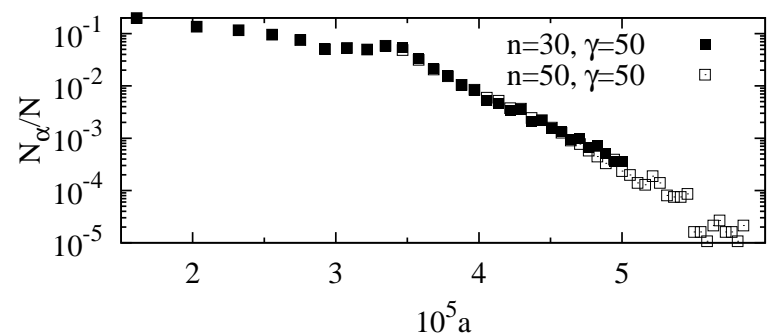

FIG. 10: Histogram of the particle size distribution in steady state for shear fragmentation for the sinusoidal shear flow for two different values of the number $n$ of size classes.

We also investigated the role of the number of size classes. As illustrated by Fig. 10, we have found that in the chosen range of $\gamma$ values the size distributions for shear fragmentation are not influenced by the fact of how many size classes have been chosen.

We also considered the dynamics of pure coagulation, without any fragmentation. This is of course a strongly non-equilibrium process in which no steady state sets in. What one observes (see Fig. 11) is that from an initially monotonically decreasing size distribution, a peak at large sizes evolves. It becomes stronger as time goes on and moves slowly towards larger size classes. Note that the large size class tail is exponential. These qualitative features turn out to be independent of the flows, and remain valid in a fluid at rest, too.

Finally we compare our findings with experiments and observations of natural phenomena. The existence of a steady state size distribution and the scaling form (10) has been observed in shear-fragmentation experiments in stirring tanks [50]. After an initial stage dominated by condensation, the distribution of cloud droplets is known to be determined by a coagulation process [9]. Field observations of cloud drop spectra show a well-separated peak at large radii which shifts in time toward larger sizes. The tail is exponential with good accuracy. 

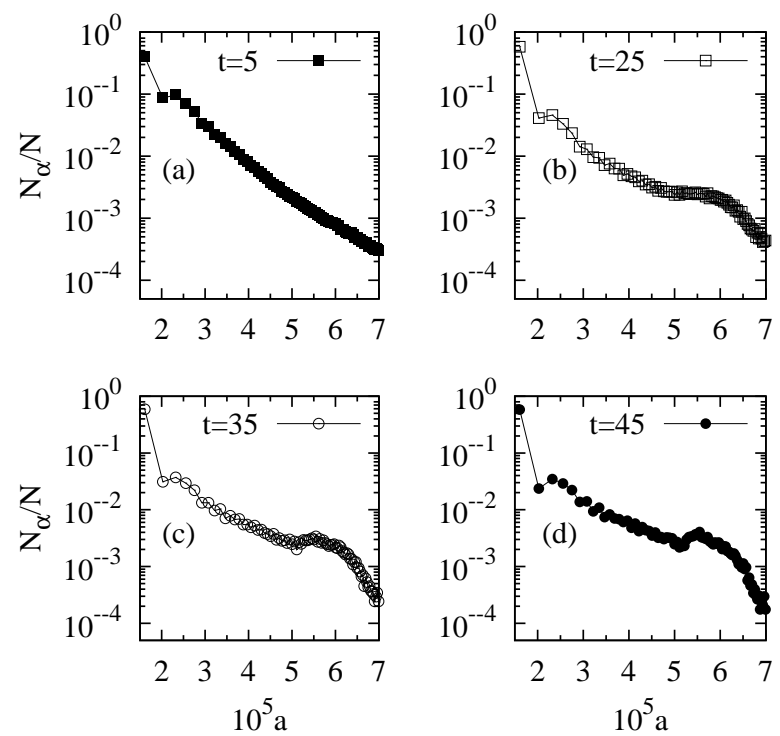

FIG. 11: Time evolution of the size distribution (snapshots taken at times $t=5,25,35,45)$ without fragmentation for the fixed convection flow, with an initial condition of $M=$ $2 \times 10^{7} m_{1}$, distributed over the first four size classes with the same mass in each of these size classes. Later instants of time are not shown because the number of particles in large size classes becomes very low.

The feature of the observed distributions that the peak clearly separates from the peak at small drop sizes might be due to the fact that collision of small and large cloud droplets are less efficient than that of comparable size droplets. This difference in efficiency is a consequence of hydrodynamical interactions, which are not taken into account in our approach. This could possibly explain why no clear separation between the two peaks in the size distribution appears in our results.

We have shown that an individual particle based modeling approach is able to reflect typical properties of coagulation and fragmentation processes of inertial particles. The appearance of a steady state is demonstrated. We outlined some of the differences in the approach to the steady state and the particle size distribution that can result from different types of fragmentation and flow. Alltogether our results suggest that the underlying flow is important to understand features of coagulatonfragmentation processes leading to steady states. However, the main features of pure coagulation, in particular the appearance of a bimodal particle size distribution, may be well captured independently of the fluid flow.

\section{ACKNOWLEDGMENTS}

The authors thank A. Alldredge, P. Franks, S. Malinowski, M. Maxey, E. Villermaux and L.-P. Wang for useful discussions and suggestions. We acknowledge the support of the Hungarian Science Foundation under the contract OTKA T72037.
[1] T. Nishikawa, Z. Toroczkai and C. Grebogi, Phys. Rev. Lett. 87, 038301 (2001).

[2] I.J. Benczik, Z. Toroczkai, and T. Tél, Phys. Rev. Lett. 89, 164501 (2002).

[3] C. Lopez, Phys. Rev. E 66, 027202 (2002).

[4] J.H.E. Cartwright, M.O. Magnasco, O. Piro and I. Tuval, Phys. Rev. Lett. 89, 264501 (2002).

[5] Y. Do and Y.C. Lai, Phys. Rev. E 70, 036203 (2004).

[6] I.J. Benczik, G. Karolyi, I. Scheuring and T. Tél, Chaos 16, 043110 (2006).

[7] R.D. Vilela and A.E. Motter, Phys. Rev. Lett. 99, 264101
(2007).

[8] G. Haller and T. Sapsis, Physica D 237, 573 (2008).

[9] R.A. Shaw, Ann. Rev. Fluid Mech. 35, 183 (2003).

[10] G. Falkovich and A. Pumir, J. Atm. Sci. 64, 4497 (2007).

[11] A. Jaczewski and S.P. Malinowski, Q. J. R. Meteorol. Soc. 131, 2047 (2005).

[12] M. Wilkinson and B. Mehlig, Europhys. Lett. 71, 186 (2005).

[13] M. Smoluchowski, Zeitschrift für physikalische Chemie, 92, 129 (1917).

[14] J. Bec, A. Celani, M. Cencini, and S. Musacchio, Phys. 
Fluids 17, 073301 (2005).

[15] E. Calzavarini, M. Cencini, D. Lohse, and F. Toschi, Phys. Rev. Lett. 101, 084504 (2008).

[16] M.R. Maxey and S. Corrsin, J. Atmos. Sci. 43, 1112 (1986).

[17] A. Fouxon, M.G. Stepanov and G. Falkovich, Nature 419, 151 (2002).

[18] L.-P. Wang, A.S. Wexler and Y. Zhou, Phys. Fluids 10, 266 (1998).

[19] J.C. Zahnow, R.D. Vilela, U. Feudel and T. Tél, Phys. Rev. E 77, 055301(R) (2008).

[20] R.O. Medrano, A. Moura, T.Tél, I.L. Caldas and C. Grebogi, Phys. Rev. E (in press).

[21] H.R. Pruppacher and J.D. Klett, Microphysics of Clouds and Precipitation, (Kluwer Academic Publishers, Dordrecht, 1997).

[22] A.L. Alldredge, T. Granata, C. Gotschalk, and T. Dickey, Limnology and Oceanography 35, 1415 (1990).

[23] P.K. Kundu, I.M. Cohen, Fluid Mechanics, Fourth Edition, Academic Press, 2008

[24] E. Villermaux, Annu. Rev. Fluid. Mech. (2008)

[25] A. Babiano, J.H.E. Cartwright, O. Piro, and A. Provenzale, Phys. Rev. Lett. 84, 5764 (2000)

[26] J. Bec, Phys. Fluids 15, L81 (2003); Phys. Fluids 17, 073301 (2005);

[27] R.W. Hockney and J.W. Eastwood, Computer Simulations using Particles, (McGraw-Hill, New York, 1981)

[28] R.D. Vilela, T. Tél, A.P.S. de Moura and C. Grebogi, Phys. Rev. E 75, 065203(R) (2007)

[29] J.C. Zahnow and U. Feudel, Phys. Rev. E 77, 026215 (2008)

[30] T. Kiorboe and G.A. Jackson, Limnology and Oceanography, 46, 1309 (2001).

[31] D.N. Thomas, S. Judd and N. Fawcett, Wat. Res. 33, No. 7, 1579 (1999).

[32] M.R. Maxey and J.J. Riley, Phys. Fluids 26, 883 (1983)

[33] T.R. Auton, J. Hunt and M. Prud'homme, J. Fluid. Mech. 197, 241 (1988)
[34] E.E. Michaelides, J. Fluids Eng. 119, 233 (1997)

[35] T. Serra and X. Casamitjana, AIChE Journal 44, 1724, (1998)

[36] J.H. Cartwright, J.M. Garcia-Ruiz, O. Piro, C.I. SainzDiaz, and I. Tuval, Phys. Rev. Lett. 93, 035502 (2004)

[37] J. Happel and H. Brenner, Low Reynolds number hydrodynamics, (Martinus Nijhoff Publishers, The Hague, 1983)

[38] I.M. Jánosi, J.O. Kessler and V.K. Horváth, Phys. Rev. E 58, 4793 (1997)

[39] G.I. Taylor, Proc. Roy. Soc. A 146, 501, (1934)

[40] M. Delichatsios, Phys. Fluids 18, 622, (1975)

[41] M.R. Maxey, Phys. Fluids 30, 1915, (1987)

[42] J. Ottino, The kinematics of mixing: stretching, chaos and transport, (Cambridge University Press, Cambridge, 1989)

[43] For the cases studied here, typical distances between particles are $\geq 50 a_{1}$.

[44] J.C. Flesch, P. Spicer and S. Pratsinis, AIChE Journal 45, 1114, (1999)

[45] A. Ben-Mizrachi, I. Procaccia and P. Grassberger, Phys. Rev. A 29, 975 (1984)

[46] S. Chandrasekhar, Hydrodynamic and Hydromagnetic Stability, (Oxford University Press, 1961)

[47] M. Liu, F. Muzzio and R. Peskin, Chaos Sol. Fract 4, 869 (1994)

[48] R.T. Pierrehumbert, Chaos Sol. Fract 4, 1091 (1994)

[49] T. Nishikawa, Z. Toroczkai, C. Grebogi and T. Tél, Phys. Rev. E 65, 020216 (2002)

[50] P.T. Spicer and S.E. Pratsinis, AIChE Journal 42, 1612, (1996)

[51] A change of the fragmentation rule has, however, important consequences. By replacing the rule of uniform distribution for the possible size classes after fragmentation by the rule that coagulates always split into two halfs of similar sizes, makes the size distribution exponential, practically over all size classes. 